Gut, 1986, 27, 1347-1352

\title{
$14 \mathrm{C}$ triolein breath test: a routine test in the gastroenterology clinic?
}

\author{
K MYLVAGANAM, P R HUDSON, A ROSS, AND C P WILLIAMS \\ From the Department of Medicine and the Department of Chemical Pathology, Wrexham Maelor Hospital, \\ Wrexham, Clwyd.
}

SUMmARY The absorption of $14 \mathrm{C}$ triolein in a standard fat meal was measured in 60 controls and 66 patients with gastrointestinal disorders by $14 \mathrm{CO}_{2}$ breath sampling. A reference range based upon cumulative eight hour values of the controls was independent of height, weight, and sex. The range was of log normal distribution and declined with age $(p<0.05)$. Acceptable 'within-day' and 'between-day' reproducibility was found. All patients tested with untreated coeliac disease, pancreatic insufficiency and most with symptomatic small intestinal Crohn's disease had subnormal values. Twenty per cent of those with irritable bowel syndrome had subnormal values. Patients with ulcerative colitis were all normal. The reagents used and the breath samples after collection were stable. In our experience the $14 \mathrm{C}$ triolein test is simple, inexpensive, and helpful in the detection of diseases associated with fat malabsorption. It is of value in monitoring the response to treatment of individual patients with coeliac disease.

Gastrointestinal symptoms such as diarrhoea, change in stools, and weight loss are among the most common complaints presenting to the general practitioner. When the patients eventually reach the physician with an interest in gastroenterology, initial investigations having been unhelpful, the question of fat malabsorption is raised. The estimation of faecal fat is traditionally regarded as the 'gold standard' for this purpose. Thorsgaard-Pederson and Halgreen have shown that, unless a controlled intake-excretion study is carried out, estimation of faecal fat is only a qualitative test. The diagnostic efficiency is only $80 \%^{1}$ and the test is widely regarded as time consuming and unpleasant for the laboratory. 'The inconvenience, the uncontrollable sources of error and the difficulties in obtaining quantitative information make measurement of faecal fat and weight inexpedient as a clinical test'. ${ }^{1}$ For these reasnns. we helieve faecal fat estimations do not meet the requirements of a district general hospital as a routine test of malabsorption. An alternative test less dependent on patient compliance is certainly needed.

The factors to be evaluated in assessing the

Address for correspondence: Dr A Ross, Consultant Physician, Wrexham Maelor Hospital, Wrexham. Clwyd LL13 7TD.

Received for publication 28 February 1986. suitability of a routine test for malabsorption have been well reviewed by Theodossi and Gazzard ${ }^{2}$ and include technical factors, biological factors (within and between subject variation), test efficiency (sensitivity, specificity plus predictive values), risks and convenience, and finally costing.

An alternative method of assessing fat malabsorption is the use of oral radio-labelled triglycerides with the subsequent measurement of breath $14 \mathrm{CO} 2$. Although this technique has been available since at least $1949,{ }^{3}$ British gastroenterologists have shown little interest in using it in clinical practice. Moreover, Chadwick ${ }^{4}$ expresses the view that there is no role for a screening test and that the $14 \mathrm{C}$ triolein test gives no assessment of the severity of fat malabsorption. Triolein labelled with $14 \mathrm{C}$ has been shown in several studies to be highly sensitive $(100 \%)$ in the detection of fat malabsorption. ${ }^{5-8}$ There are several problems in judging the place of this type of test, in particular, many variations in protocol have been reported on small numbers of patients and controls. The reproducibility of the procedure has not been established and hence its usefulness in following the progress of individual patients is not known. In a valuable study, West et al ${ }^{6}$ using a standard $60 \mathrm{~g}$ fat meal as a carrier for $14 \mathrm{C}$ triolein, tested eight control subjects and 52 patients with various gastrointestinal diseases. Reproducibility studies and 
follow up measurements were not reported. This study, nevertheless, further illustrates the potential of the $14 \mathrm{C}$ triolein test as a routine procedure. We adopted West's method in order to produce comparative data. As the sensitivity of the $14 \mathrm{C}$ triolein absorption test in detecting fat malabsorption has already been established for this protocol, we have not regarded it as obligatory to couple our study with faecal fat estimations.

In our study we set out to answer the following questions: (1) Is the test a practical proposition for routine use, in particular are the reagents and breath samples stable? (2) What is the reference range, and is it affected by age, height, weight or sex? (3) Is the test reproducible? (Theodossi and Gazzard ${ }^{2}$ have observed that within-subject variability has probably only been determined for faecal fats). (4) Is the test valuable in detecting gastrointestinal diseases which have caused malabsorption? (5) Is the test of use in monitoring the response to treatment?

\section{Methods}

\section{SUBJECTS}

Tests were conducted with the informed consent of the subjects and with written permission from the hospital ethical committee. One hundred and twenty six subjects were studied: 60 controls and 66 patients with various gastrointestinal diseases. In the control group there were 42 women and 18 men with an age range of 17-87 years drawn from the hospital inpatients and outpatients. The 66 patients with gastrointestinal disease included 10 with coeliac disease, 15 with Crohn's disease, 31 with irritable bowel syndrome, seven with ulcerative colitis and three with pancreatic insufficiency. All but one patient completed the test. None of the subjects studied had any disorders such as chronic respiratory disease, hyperthyroidism or liver disease which may have affected the test values.

Five microcurie $(0 \cdot 185 \mathrm{MBq})$ of $14 \mathrm{C}$ triolein (Amersham International plc, White Lion Road, Amersham, HP7 9LL) dissolved in $50 \mu \mathrm{l}$ of toluene, supplied by Amersham, was added on the morning of the test to the $60 \mathrm{gram}$ meal as formulated by West $e t a l^{6}$ (excluding the buttered toast or bread). The toluene was allowed to evaporate before mixing the meal. The amount of radioactive triolein was half that used by West. The meals were prepared in the hospital kitchen the previous day and stored in a domestic refrigerator. Subjects were fasted overnight and the test meal administered. Duplicate breath samples were collected before the meal and at hourly intervals thereafter for eight hours. Subjects were allowed to drink unsweetened black tea, coffee, or water during the test and asked not to smoke after the first hour.

Approximately $1 \mathrm{mmol} \mathrm{CO} 2$ was collected in $2 \mathrm{ml}$ trapping reagent containing phenolphthalein $(0.02 \% \mathrm{w} / \mathrm{v})$ as indicator in equal volumes of 'hyamine 10×hydroxide solution' (BDH Chemicals, Broom Road, Poole, BH12 4NN) and ethanol. The $\mathrm{CO} 2$ was collected by blowing through $150 \mathrm{~mm}$ disposable porous plugged Pasteur pipettes (Bilbate Ltd, 10 Low March, Daventry, North Hants, NN11 4PQ). Subjects blew directly into the trapping solution contained in a $20 \mathrm{ml}$ scintillation vial using a new pipette for each pair of vials to avoid water/ saliva droplets entering the vials. If this occurred, an opalescent solution formed which interfered with the radioactive measurements. The opalescence could be reversed by adding $0.5 \mathrm{ml}$ ethanol. Observations were made on the stability of the trapping reagent and the stability of the mixture containing the exhaled carbon dioxide before addition of the scintillation solution. Two separate sets of results were calculated from the two vials collected each time breath was sampled. From the individual hourly values $\left(\%\right.$ dose $\left./ \mathrm{mmol} \mathrm{CO} 2 \times 10^{4}\right)$ an eight hour cumulative result was calculated ${ }^{6}$ for each set of vials as a test of 'within-day' reproducibility. The mean of these two was taken as the final result. Tests were repeated in some controls after a minimum six month interval to check the reproducibility over time ('between-day'). Subjects conducted the tests in groups of three to five in a side room, normally on an outpatient basis.

\section{Results}

STABILITY OF THE REAGENTS

No change was found at room temperature in titratable alkalinity over a three month period in capped collection vials containing $2 \mathrm{ml}$ trapping reagent prepared for patient use.

\section{STABILITY OF COLLECTED SAMPLES}

Slight pinkness reappearing in the trapping reagent a few minutes after completion of the breath collection in vials was of concern initially. From titration experiments, however, the apparent loss of $\mathrm{CO} 2$ from capped vials as completed by the patients did not exceed $3 \%$ when followed at intervals up to three weeks.

\section{REFERENCE RANGE}

The distribution of eight hour cumulative values from the 60 controls had a positive skew (mean 306, SD 58, coefficient of skewness $0 \cdot 66$ ). Logarithmic transformation reduced the coefficient of skewness to $0 \cdot 24$, so a reference range of $207-435$ (95\% 
confidence limits) was calculated using the transformed data.

Multiple regression showed a significant fall in the cumulative eight hour value with age $(p<0 \cdot 05)$, but no change with height, weight or sex.

Simple regression of the cumulative eight hour value against age gave the following regression equation:

eight hour value $=366.5(+/-48 \cdot 14)-1.26(+/-$ $0.95) \times$ age in years (values in parentheses are the $95 \%$ confidence limits) Correlation coefficient $=$ $-0.33(\mathrm{p}<0.05)$.

This corresponds to a fall of $20 \%$ between the ages of 17 and 70 years. We did not apply this correction to the interpretation of the results in patients because there were insufficient observations in each control group when divided by decades.

\section{WITHIN-DAY REPRODUCIBILITY}

The actual separately calculated eight hour values never differed from the mean by more than $2 \%$. There was no significant difference between these two sets of results by paired $t$ test.

BETWEEN-DAY REPRODUCIBILITY (Fig. 1)

The figure illustrates the results in nine controls who agreed to have their test repeated after an interval of 6-12 months. In all subjects, results of the second test were within $21 \%$ (mean $8.5 \%$, SD 5.4 ) of the first test.

PATIENTS With COEliac Disease (Fig. 2) All with histologically confirmed coeliac disease

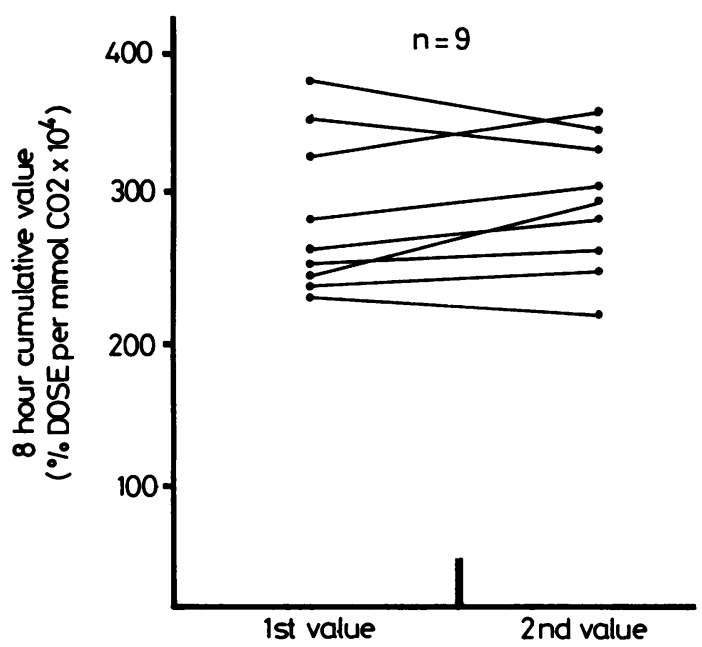

Fig. 1 First and second values with an interval of six to 12 months in nine control subjects.

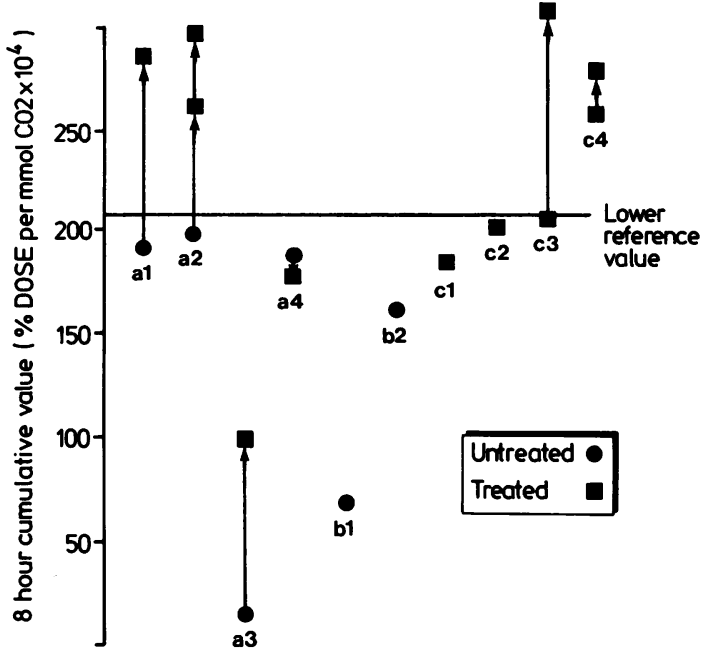

Fig. 2 Results in 10 patients with coeliac disease. Direction of arrows indicates the chronological order where tests were repeated.

had subnormal values when seen before treatment (patient a1-4 and b1-2). Four patients had a 14C test and a repeat biopsy six months after starting their gluten free diets (patients a1-4). Patients a1, $\mathrm{a} 2$, and $\mathrm{a} 3$ showed an improvement in absorption, while the change in patient a4 was negligible. Repeat jejunal biopsies in patients a1 and a 2 were normal. Partial histological remission was noted in patient a3. No remission had occurred in patient a4 Repeat tests were not done on patients b1 and b2. Patients c1, c2, c3, and c4 were already on gluten free diets at the time the tests were done. Repeat $14 \mathrm{C}$ triolen tests after six months in patients $\mathrm{c} 3$ and c4 showed improvement in absorption values.

PATIENTS WITH CROHN'S DISEASE (Fig. 3) Of the 11 with small intestinal involvement, as judged by barium studies, all but one with symptoms (diarrhoea with or without abdominal pain) had subnormal results. Patients with only large intestinal involvement had normal values whether they were symptomatic or not. Test results improved in all six who became asymptomatic.

PATIENTS WITH IRRITABLE BOWEL SYNDROME

(Fig. 4)

Of the 31 , one did not complete the test. Of the rest, 25 were symptomatic and five were asymptomatic at the time of the test. Six in the symptomatic group had subnormal values. Jejunal biopsy was carried out in three of these patients and normal histology was seen. The other three refused to have the test 


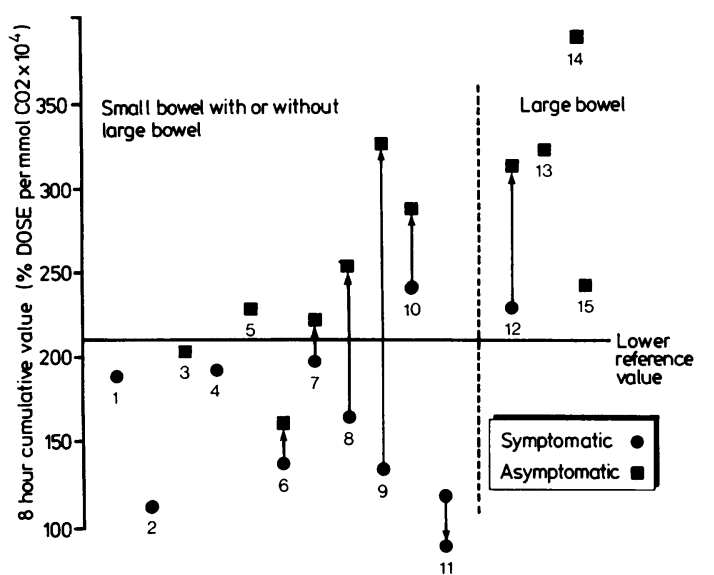

Fig. 3 Results in 15 patients with Crohn's disease. Direction of arrows indicate the chronological order where tests were repeated.

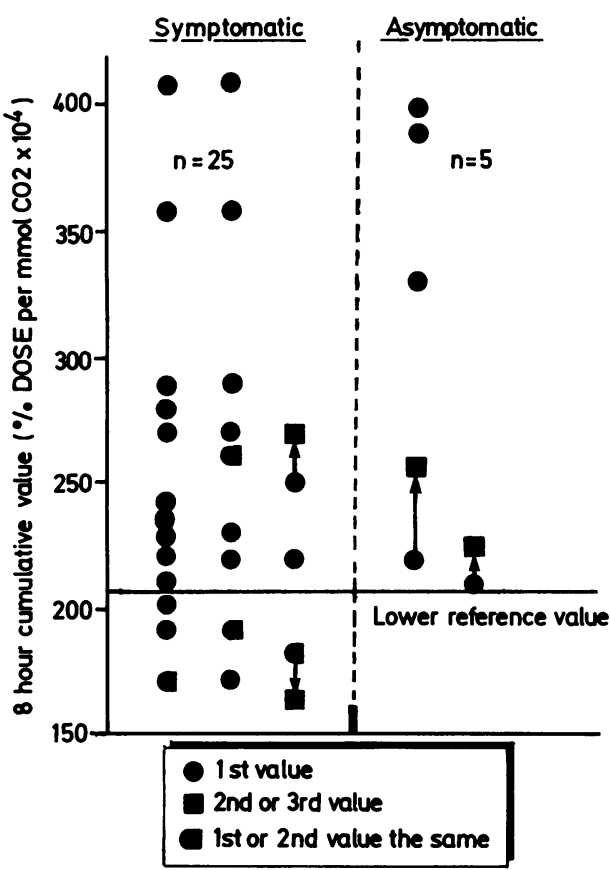

Fig. 4 Results in 30 patients with the irritable bowel syndrome. Direction of arrows indicates the chronological order where tests were repeated.

done. Seven patients had the test repeated after 6-12 months. There was no change in the symptomatology of these and the results of the second test in all but one were within $8 \%$ of the first, the exception being $17 \%$.
PATIENTS WITH PANCREATIC INSUFFICIENCY

There were two with untreated pancreatic insufficiency who had values of 9 and 34 which increased respectively to 118 and 125 one month after starting Pancrex. Six months later a result of 134 was recorded from the second patient. Another with known pancreatic insufficiency who had been established on Pancrex for five years and was completely symptom free, had a normal value of 351 .

\section{PATIENTS WITH ULCERATIVE COLITIS}

All seven in this group had normal results.

\section{Discussion}

The authors and the subjects found the test remarkably easy to complete. Although $60 \mathrm{~g}$ fat is unphysiologically large for a single meal, it can be argued that a discriminatory advantage may be obtained by stressing the absorptive capacity. The majority did not find the meal unpleasant and were not distressed by afternoon hunger. We found it an advantage to provide subjects with a detailed instruction sheet in advance of the test. These instructions also contained an explanation of the principle of the test which was repeated during a 20 minute period of instruction at the beginning of the test. Subjects thereafter, looked after themselves under the general supervision of a senior nurse. It would be practicable to complete the breath collections at home, particularly as we have noted that there is no technical necessity to process the samples on the same day. A measure of the acceptability of the test procedure was that subjects did not object to repeat examinations.

In faecal fat estimations the problems of collecting specimens outweigh all other errors, ${ }^{2}$ but this is not the case with $14 \mathrm{C}$ breath collections. We were impressed by the 'within-day' reproducibility of the tests and had anticipated greater variability of duplicate samples. The explanation can be found in studies by Kaihara and Wagner ${ }^{9}$ who showed that it was only necessary for the patient to decolourise (neutralise) the trapping solution and that further bubbling through the solution did not result in the accumulation of further radioactive counts. It is possible to decolourise the trapping agent in less than half a minute, but some found blowing through the porous plugs of the pipettes strenuous and took as long as five minutes. Removal of the wool plug would minimise this problem, but at least two patients sucked up trapping agent and were protected by the plugs. As an additional precaution, patients now have $15 \mathrm{~cm}$ plastic tubing between their mouth and the tube. 
One problem of introducing this type of test is establishing a reference range. We therefore closely followed the protocol of West et at in order to benefit from their experience. Our lower reference value of 207 for eight hours is in general agreement with their findings allowing for the larger population in our study. The $20 \%$ decline in mean values with age was not weighted by extreme old age, as all but one of the controls were 70 years old or less. This indicates that further work should be done on age related change, although Strange et $a^{10}$ reported no improvement in the discriminatory capacity of a 14C labelled tripalmitate test when correcting for age.

The merits of any procedure must be balanced against its cost. The test requires a beta radiation counter which may not be available in every district general hospital. This could be shared between several hospitals as the stability of the samples allows appropriate transport for counting. Even allowing for capital depreciation on a counter solely dedicated to the test, our cost per patient, for 200 patients per annum, does not exceed that of two chest radiographs.

Is a simple, reliable and economical test for fat malabsorption needed by the clinician? In a busy district general hospital, where large numbers of unselected patients present for assessment, our experience is that the test does help to orientate further investigations to a level proximal to the ileocaecal valve. Barium studies of the small intestine are still being used to screen for malabsorption, with many false positives. Biopsy of the small bowel mucosa, whether under fluoroscopy or via an endoscope is an invasive procedure and cannot be regarded as a screening test.

All our patients with untreated coeliac disease had subnormal values which indicate that the test is helpful in screening for this condition. Intestinal biopsy is, of course, mandatory to establish the diagnosis. An increase in absolute absorption in individual patients reflected histological remission. We believe, therefore, that the test offers some quantitative measure of response to treatment in the individual patient, which is helpful if a follow up biopsy is refused.

Subnormal fat absorption, in the absence of liver disease and pancreatic insufficiency is an indication of small intestinal pathology. All but one of our patients with symptomatic Crohn's disease of the small bowel had abnormal values, even when only the terminal ileum appeared diseased on barium studies. In all of the symptomatic Crohn's patients who had the test repeated when they were asymptomatic, an increase in absolute absorption was noted. The very tentative conclusion is therefore, that for an individual patient with Crohn's disease, absorption values vary inversely with symptoms, which possibly reflect activity. This test is not helpful as a screening test for Crohn's disease in view of the proportion of negative results. A subnormal value, however, should raise the suspicion of Crohn's disease in patients with nonspecific gastrointestinal complaints.

The findings in patients diagnosed as having irritable bowel syndrome are interesting. Twenty per cent of our patients had subnormal values which is consistent with the findings of Newcomer et al. ${ }^{5}$ An appreciable number of false positive tests would not be surprising due to the low specificity of the test. ${ }^{5-8}$ We have not studied these patients further, apart from excluding coeliac disease in those who permitted a biopsy. It is always possible that unrecognised gastrointestinal pathology existed. It may also be possible that these patients have bile salt malabsorption, as described by Merrick et al ${ }^{11}$ and before them by Thaysen and Pedersen. ${ }^{12}$ In any event, the finding of a low value should stimulate more detailed assessment, in the hope of finding a treatable condition. All patients with ulcerative colitis had normal values as would be anticipated since fat malabsorption is not a feature of this condition.

The $14 \mathrm{C}$ triolein fat absorption test has now become part of our routine workup for patients with unexplained bowel disturbance, weight loss and worries about their bowels. A normal value gives a measure of reassurance to both physician and patient about small intestinal function, although it in no way excludes gastrointestinal disease. A low value indicates the need for more expensive and unpleasant investigations. In patients with proven coeliac disease, the test has proved helpful in following the response to treatment.

This study was not prospective in the true sense of the word and the value of this test in consecutive patients presenting to a clinic with gastrointestinal symptoms therefore still remains to be established.

We are grateful to Miss C J Sorenson, Miss P A Cowley (Chief Dietitians) and Mr P D Dempsey (Catering Manager) for the fat meals and to Miss W Savage (Senior Nurse) for the supervision of patients during tests. We are also grateful to Mrs J F Jones and Miss P A Davies for secretarial and administrative help.

\section{References}

1 Thorsgaard Pedersen N, Halgreen H. Faecal fat and faecal weight - reproducibility and diagnostic efficiency of various regimens. Scand J Gastroenterol 1984; 19: 350-4. 
2 Theodossi A, Gazzard BG. Have chemical tests a role in diagnosing malabsorption? Ann Clin Biochem 1984; 21: $153-65$.

3 Stanley MM, Thannhauser SJ. The absorption and disposition of orally administered 1131 labelled neutral fat in man. $J$ Lab Clin Med 1949; 34: 1634-39.

4 Chadwick VS. Clinical investigation of patients with malabsorption and diarrhoea. In: Bouchier IAD. Allan RN, Hodgson HJF, Keighley MRB, eds. Textbook of gastroenterology. London: Bailliere Tindall, 1984: 438.

5 Newcomer AD, Hofmann AF, Dimagno EP. Thomas PJ, Carlson GL. Triolein breath test. A sensitive and specific test for fat malabsorption. Gastroenterology 1979; 76: 6-13.

6 West PS, Levin GE, Griffin GE, Maxwell JD. Comparison of simple screening tests for fat malabsorption. Br Med J 1981; 282: 1501-4.

7 Einarsson K, Björkhem I, Eklöf R, Blomstrand R.
14C-Triolein breath test as a rapid and convenient screening test for fat malabsorption. Scand J Gastroenterol 1983; 18: 9-12.

8 Butler RN, Lawson MJ, Gehling NJ, Kerr Grant A. Clinical evaluation of the 14C-triolein breath test: a Critical Analysis. Aust N Z J Med 1984; 14: 111-13.

9 Kaihara S,Wagner HN. Measurement of intestinal fat absorption with carbon-14 labelled tracers. J Lab Clin Med 1968; 71: 400-11.

10 Strange RC, Reid J, Holton D, Jewell NP, Percy-Robb IW. The glyceryl (14C) tripalmitate breath test: A reassessment. Clin Chim Acta 1980; 103: 317-23.

11 Merrick MV, Eastwood MA, Ford MJ. Is bile acid malabsorption underdiagnosed? An evaluation of diagnosis by measurement of SeHCAT retention. $\mathrm{Br}$ Med J 1985; 290: 665-8.

12 Thaysen EH, Pedersen L. Idiopathic bile acid catharsis. Gut 1976; 17: 965-70. 